Document downloaded from:

http://hdl.handle.net/10251/177802

This paper must be cited as:

Abderrazak, F.; Antonino Daviu, E.; Ferrando Bataller, M.; Taldi, L. (2020). Analysis of Magnetically-Coupled Loops Based On Characteristic Modes. IEEE. 121-122. https://doi.org/10.1109/IEEECONF35879.2020.9329736

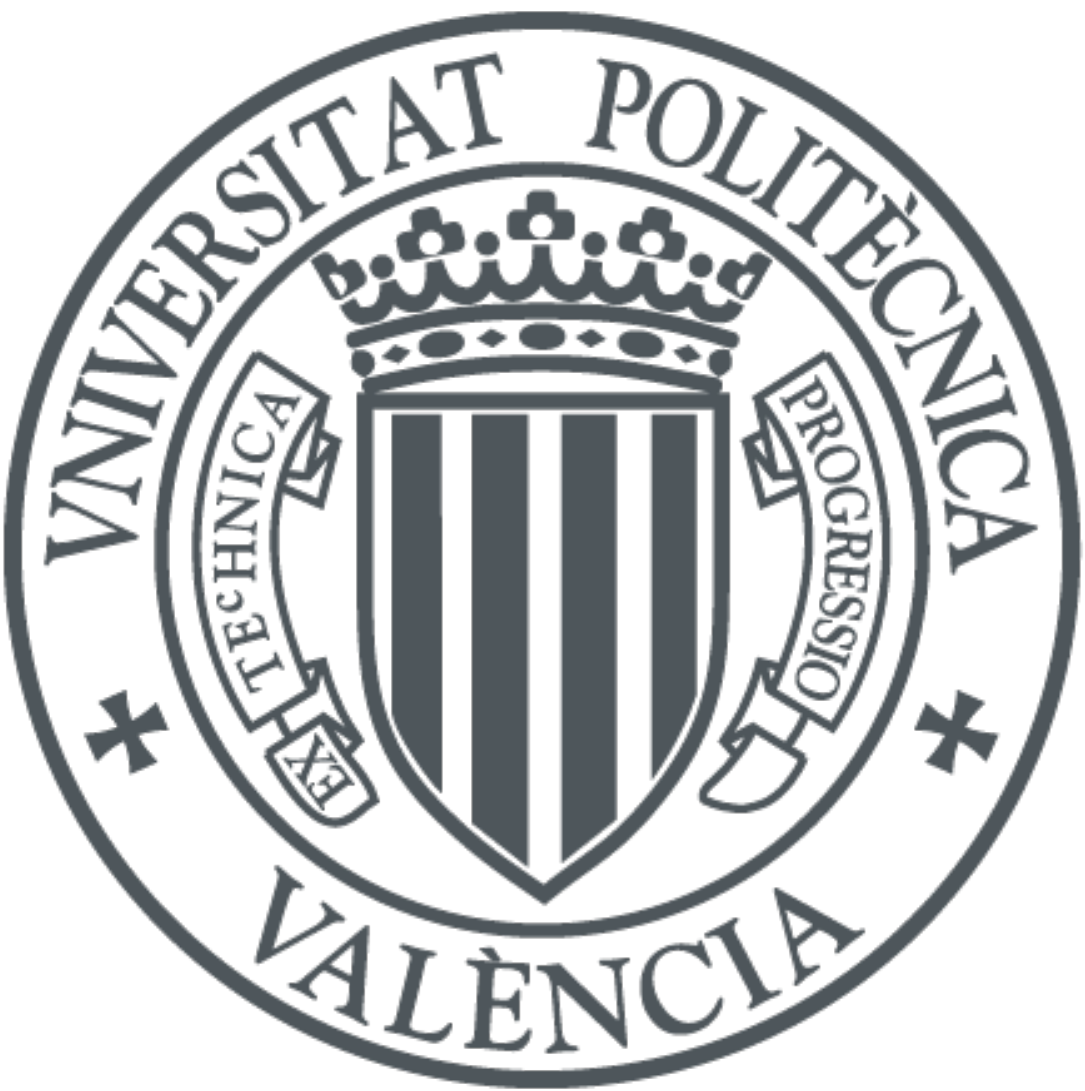

The final publication is available at

https://doi.org/10.1109/IEEECONF35879.2020.9329736

Copyright IEEE

Additional Information 


\title{
Analysis of Magnetically-Coupled Loops Based On Characteristic Modes
}

\author{
Ferdaous Abderrazak ${ }^{1,2}$, Eva Antonino-Daviu ${ }^{1}$, Miguel Ferrando-Bataller ${ }^{1}$, Larbi Talbi ${ }^{2}$ \\ ${ }^{1}$ Instituto de Telecomunicaciones y Aplicaciones Multimedia (iTEAM), Universitat Politècnica de València \\ Camino de Vera, s/n, 46022 Valencia, Spain, e-mail: ferab@iteam.upv.es \\ ${ }^{2}$ Electrical Engineering Department, University of Quebec in Outaouais, Gatineau, Quebec, Canada
}

\begin{abstract}
A preliminary numerical magnetic field study of a wireless power transfer system composed of two interconnected source loops is addressed in this paper. The key feature of this study is to propose a new configuration capable of maintaining a uniform magnetic field density between the two loops. Furthermore, it has been shown that the Theory of Characteristic Modes is a helpful tool for investigating the near field of the magnetically-coupled loops.
\end{abstract}

Keywords-Characteristic Mode, Loop Antenna, Magnetic Flux density, Near Field Analysis, Medical WPT System, Wireless Charging.

\section{INTRODUCTION}

Wireless Power Transfer (WPT) systems are providing great efficiency, utility and safety where wired power transmission has some difficulties or hazardous technical problems [1]. In this context, new features, such as powering micro medical robots, can be achieved. Recently, Near Field (NF) coupling based on a multi-transmitter WPT system for implementable cardiac pacemaker, operating in the Industrial, Scientific and Medical (ISM) frequency band, has demonstrated an affective charging power of $5 \mathrm{~W}$ [1]. It is worth underlining that this system is efficient only when the patient is stable. However, the authors did not take into consideration the sensitivity of the magnetic field between the transmitting coils. Indeed, the Theory of Characteristic Mode (TCM) has shown its utlity in providing physical insight on the coupling, and describing the misalignment sensitivity between two parallel loops [2].

With regard to the given solution in [1], this paper focuses on proposing a new WPT link consisting in interconnecting two transmitting loop antennas. This design maintains the stability of the magnetic field to lateral sensitivity. The proposed system can be employed to charge the battery of micro medical robotics such as cardiac pacemaker through magnetic coupling (as shown in Fig. 1) at $403 \mathrm{MHz}$ in the MedRadio Service core band. Using the Electromagnetic Simulation Software FEKO, the loops are analyzed from the TCM point of view, considering the current distributions of Characteristic Modes (CMs), and their magnetic field density.

\section{Modal Analysis of THE Two o InTERCONNECTED LOOPS}

\section{A. Concise Description of the TCM}

Based on the method of moments, and using the Rao Wilton Glisson basis functions, the TCM generates the diagonalization of the impedance matrix $Z$ through the weighted eigenvalue equation given by [3]:

$$
X\left(J_{n}\right)=\lambda_{n} R\left(J_{n}\right)
$$

where $\lambda_{n}$ are the eigenvalues, $J_{n}$ are the eigencurrents, and $R$ and $X$ are the real and the imaginary part of $Z$, respectively. The resonance of each $\mathrm{CM}$ can be defined when its eigenvalue is equal to zero. Considering this resonance condition, the mode resonates when its Modal Angle $\left(M A_{n}\right)$, illustrated in (2), is equal to $180^{\circ}$ :

$$
M A_{n}=180^{\circ}-\arctan \left(\lambda_{n}\right)
$$

The half-power radiating bandwidth is defined when the modal significance $\left(M S_{n}\right)$, given by (3), is above 0.7 :

$$
M S_{n}=\frac{1}{\left|1+j \lambda_{n}\right|}
$$

A CM Analysis (CMA) of the magnetically-coupled loop antennas is performed, focusing on investigating the stability and the intensity of the modal and the total magnetic fields.

\section{B. CMA of Two Magnetically-coupled Loop Antennas in the $N F$}

A WPT system consisting in interconnecting two identical transmitting metallic loops of radius $r$ equal to $27.75 \mathrm{~mm}$ and a wire width $w$ of $8 \mathrm{~mm}$, is analyzed using a CMA from 0.3 to 1 $\mathrm{GHz}$. The receiver is placed between the two loop antennas, which are interconnected through two vertical wires of length (d) equal to $190 \mathrm{~mm}$, and the receiver is placed on between as shown in Fig. 1. Indeed, the efficiency of the WPT can reach a distance equal to eight times the radius of the wire [3]. Fig. 2 provides the $M A_{n}$ and the $M S_{n}$ of the different CMs over the indicated frequency band. To better describe the modes, their currents distributions and magnetic fields are given in Fig. 3.

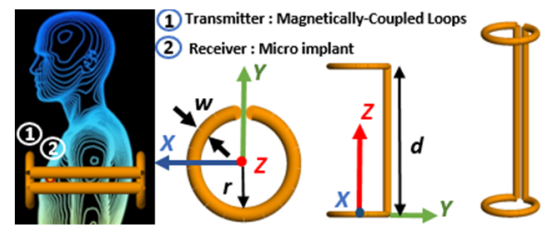

Fig. 1. The proposed medical WPT system and its geometry. 


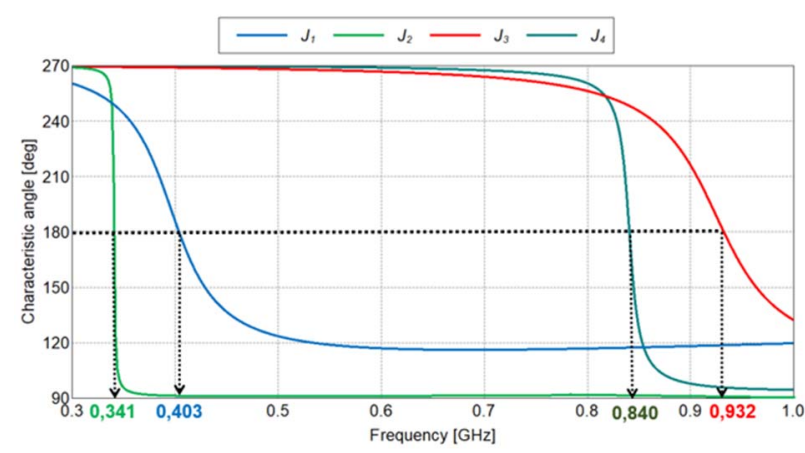

(a)

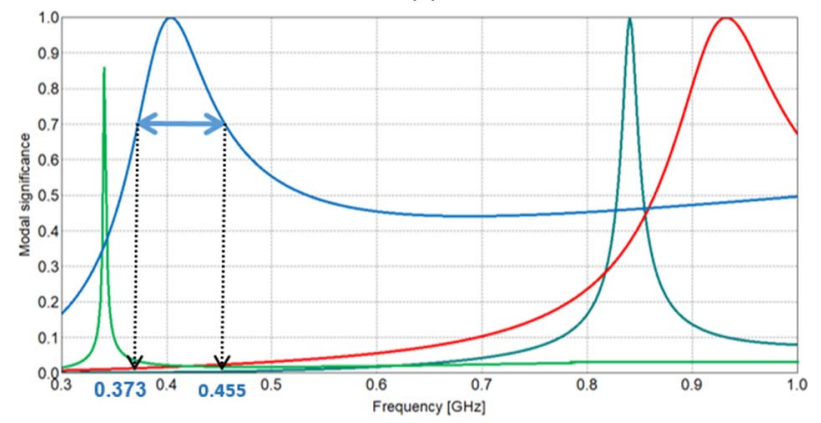

(b)

Fig. 2. CMA of the magnetically-coupled loop antennas: (a) Characteristic Angle, (b) Modal Significance.

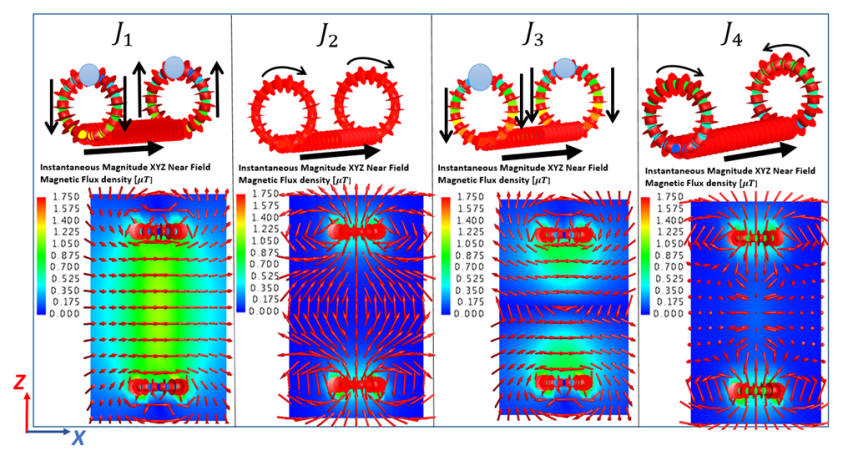

Fig. 3. Current distributions and magnetic field in the $\mathrm{XZ}$ surface $(\mathrm{Y}=0)$ of each $\mathrm{CM}$ at its resonant frequency.

CMs are classified in Antenna Mode (AM) and Transmission Line Mode (TLM) according to their current flowing directions. The AM is defined when the currents of the two wires flow in phase. Conversely, when the currents flow with $180^{\circ}$ difference, the mode is called a TLM [3]. Indeed, $J_{l}$, which is a TLM resonating at $403 \mathrm{MHz}$, presents a magnetic flux density of $1.225 \mu \mathrm{T}$ between the two loops. It is the most stable and concentrated field density compared to other CMs. Its associated $\mathrm{AM} J_{3}$, which resonates at $932 \mathrm{MHz}$, reaches a maximum of $1.105 \mu \mathrm{T}$ around the loops, and, it is cancelled in the region in midst. The two loop modes $J_{2}$ and $J_{4}$ present a null magnetic field between the transmitters.

The AM has been always considered as a better radiator with a broader bandwidth compared to the TLM since the current in both arms are flowing in phase. Yet, recent research on WPT between two parallel loops has shown that the TLM is the mode contributing mostly to the power transfer efficiency [2]. Besides, the simulation results, shown in Fig. 2 (b), evidence that the TLM $J_{l}$ provides a half-power radiating bandwidth in the frequency range from $373 \mathrm{MHz}$ to $455 \mathrm{MHz}$, which covers the MedRadio frequency band $(401-406 \mathrm{MHz})$. Yet, in order to enhance the excitation of $J_{l}$, two ports are placed in the two horizontal links as shown in Fig. 4.

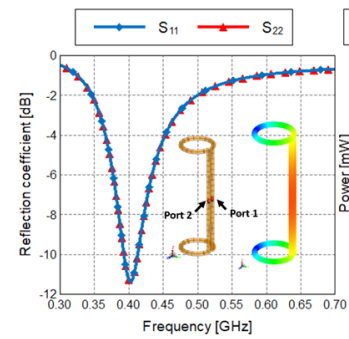

(a)

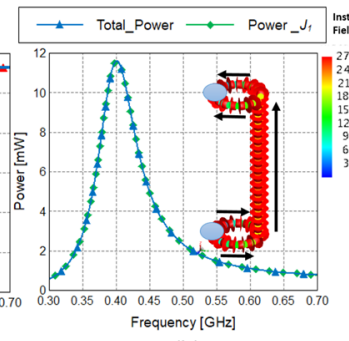

(b)

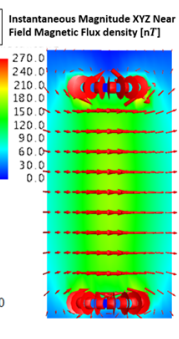

(c)
Fig. 4. Enhancing the excitation of $J_{l}$ : : (a) Reflexion coefficients, (b) Total power, the power provided by $J_{l}$ and the current distribution at resonant frequency, (c) Magnetic field at resonance in the $\mathrm{XZ}$ surface $(\mathrm{Y}=0)$.

The TLM $J_{1}$ is fully contributing to the total power of the system along the frequency band. At resonant frequency, 403 $\mathrm{MHz}$, the minimum of the reflection coefficients is $-11.38 \mathrm{~dB}$. Moreover, the observed mode at resonance is an odd mode, which is a TLM when drawing an analogy with the TCM. A very stable and concentrated magnetic field reaching a density of 240 $\mathrm{nT}$ is observed between the two transmitting loop antennas.

\section{CONCLUSION}

The Theory of Characteristic is showing a high capability in providing physical insight into magnetic coupling between loop antennas. Indeed, although the excitation of the Transmission Line Mode (TLM) has been always avoided, the results provided by this study demonstrate that it yields the strongest and the most concentrated magnetic field between two magnetic resonators independently on the lateral alignment compared to other Characteristic Modes. Further studies taking into account the sensitivity of the magnetic field to an effective homogenous medium placed between the loop antennas will be presented at the conference.

\section{REFERENCES}

[1] C. Liu, C. Jiang, J. Song, K. T. Chau, "An effective sandwiched wireless power transfer system for charging implantable cardiac pacemaker," in IEEE Transactions on Industrial Electronics, vol. 66, no 5, pp. 4108-4117, May 2019.

[2] F. Abderrazak, E. Antonino-Daviu, M. Ferrando-Bataller, "Power Transfer Efficiency Analyzed using Characteristic Mode Coupling Between Two Parallel Loops," Accepted for publication in the European Conference on Antenna and Propagation 2020 (EUCAP2020).

[3] A. Kurs, A. Karalis, R. Moffatt, J. D. Joannopoulos, P. Fisher and M. Soljac, "Wireless power transfer via strongly coupled magnetic resonances," in Science Magazine, vol. 317, no 5834, p. 83-86, July 2007. 